\title{
Homologous Series of Flower Phases in Metal-Organic Networks on Au(111) Surface
}

\author{
Anastasiia I. Fadeeva,* Vitaly A. Gorbunov, Olga S. Solovyeva, \\ Pavel V. Stishenko, Alexander V. Myshlyavtsev \\ Omsk State Technical University, 11 Mira, Omsk, 644050, Russian Federation \\ *nastya-fad@mail.ru
}

\section{Analysis of the model ground state}

Two phase sequences are formed in the lattice model of the TPyB$\mathrm{Me} / \mathrm{Au}(111)$ adsorption layer. The first one appears at higher chemical potentials (or low partial pressures) of the metal. We call it "the upper sequence". The second one is realized at lower chemical potentials (or higher partial pressures) of the metal. It is "the lower sequence".

The structural elements of the phases from the lower sequence (Figure 1S) can be related to the pentagons and rhombuses observed in the STM experiment [1]. These phases are also formed in our evolutionary search of the structures and the Monte Carlo simulation at nonzero temperatures. However, in the main text we discuss in details only the upper sequence of the phases, because these phases are observed in several metal-organic layers and, therefore, have a general interest. 
We have derived a formula for the grand potential $\Omega$ for the each phases of both phase sequences. Here, the grand potential is a function of the chemical potential of each component in the adayer $\left(\mu_{T P y B}, \mu_{M e}\right)$ and the intermolecular interaction energies - the TPyB-Me coordination $(w)$ and weak repulsion between pair of neighboring TPyB molecules $\left(w_{T P y B}\right)$ :

$$
\begin{aligned}
& \Omega_{n=i}=\frac{n(n+2) \cdot \mu_{M e}+n(n+1) \cdot \mu_{T P y B}+3 n(n+1) \cdot w+3 n(n-1) \cdot w_{T P y B}}{3(n+1)^{2}} \\
& \Omega_{n^{\prime}=i} \\
& =\frac{n^{\prime}\left(n^{\prime}+2\right) \cdot \mu_{M e}+n^{\prime}\left(n^{\prime}+1\right) \cdot \mu_{T P y B}+3 n^{\prime}\left(n^{\prime}-1\right) \cdot w+\left(3 n^{\prime 2}-3 n^{\prime}+2\right) \cdot w_{T P y B}}{3 n^{\prime}\left(n^{\prime}+2\right)}
\end{aligned}
$$

Where $n$ and $n^{\prime}$ are the sequence number of the phase in the upper and lower phase sequences, respectively.

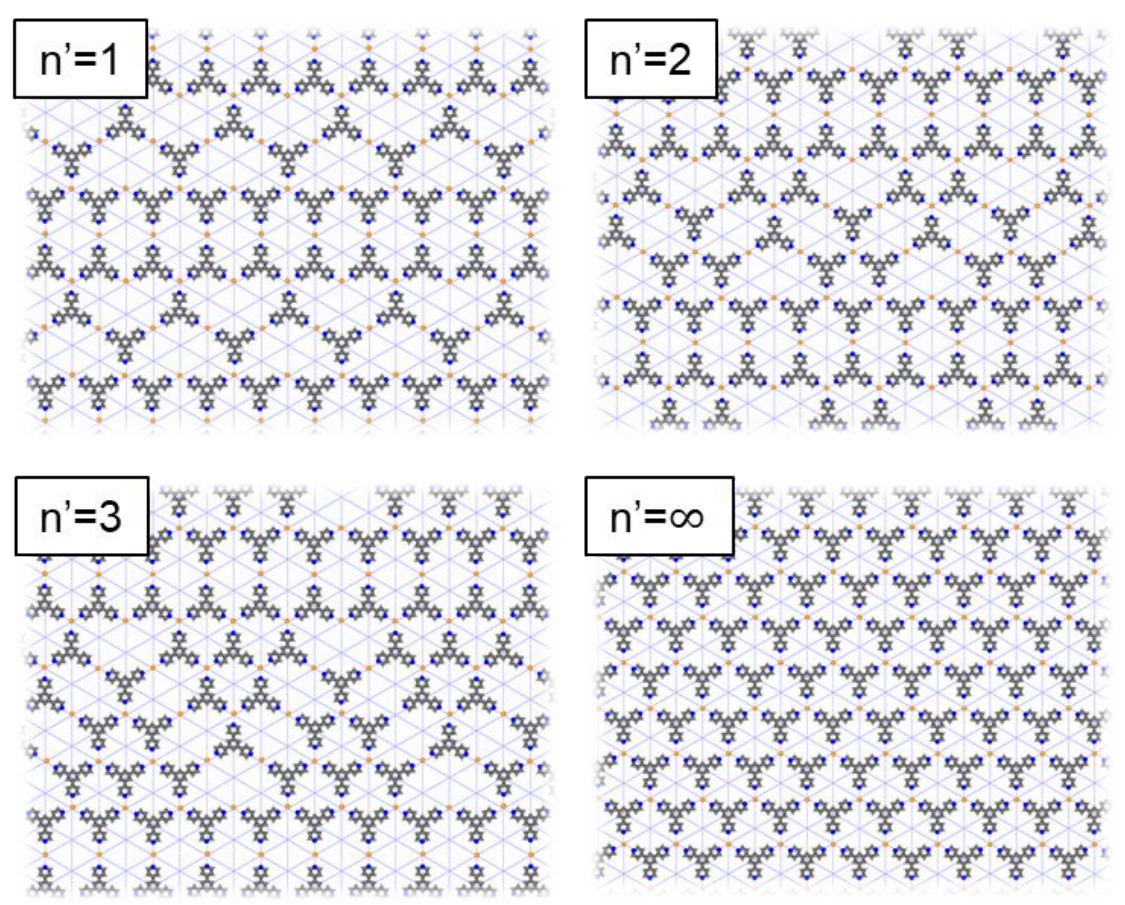

Figure S1. Lattice models of ordered metal-organic structures in the lower sequence of the TPyB-Me/Au(111) adsorption layer. 
To determine which phase exists at a $\left(\mu_{T P y B}, \mu_{M e}\right)$ point for the fixed $w$ and $w_{T P y B}$, we used the following principle. If the grand potential of the $i$ phase is less than that of all other $j$ phases $\Omega_{i}<\Omega_{j \neq i}$, then $i$ phase is thermodynamically favorable phase at these conditions. The ground state phase diagram of the TPyB$\mathrm{Me} / \mathrm{Au}(111)$ lattice model including two phase sequences is shown in Figure 2.

The left edge point between two sequences (Figure S2) in terms of TPyB chemical potential is determined by the expression:

$$
\mu_{M e} /|w|=\frac{15}{4} w_{T P y B}
$$

This point is the intersection of the line corresponding to $n=n '=\infty$ phase and the line of $n=(\infty-1)$ and $n^{\prime}=(\infty-1)$ phases coexistence. If the chemical potential of the metal is lower than this value, the phases from the lower sequence can appear, if not - the upper phase sequence is realized.

The right boundary between the two phase sequences is $\mu_{M e} /|w|=0$ (Figure S2). It is the intersection of the coexistence lines of the $n=2 / n^{\prime}=2$ phases and the $n^{\prime}=1 / n^{\prime}=2$ phases. Thus, at negative chemical potentials of the metal (at relatively high partial pressures of the metal), the upper sequence of the phase does not appear in the metal-organic layer. Only exception is the honeycomb structure that always stable at low partial pressures of the TPyB. 


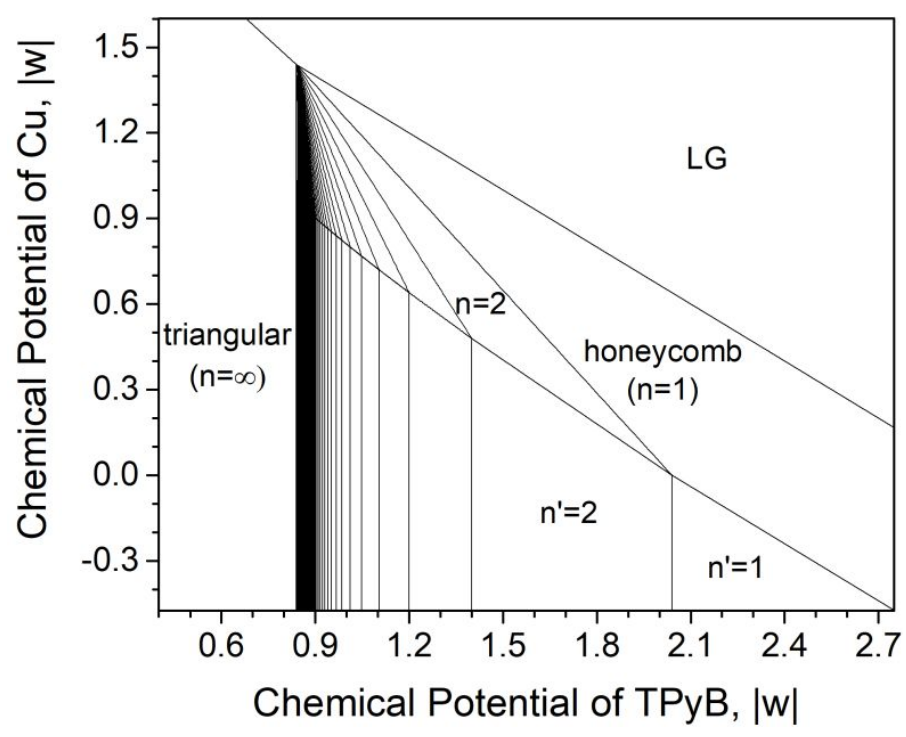

Figure S2. Phase diagram of the lattice model of the TPyB-Me/Au(111) adsorption layer with two sequences in the $\left(\mu_{T P y B}, \mu_{C u}\right)$ coordinates: $w / \mid w$ $\mid=-1.0, w_{t} / w_{d}=1.14$ (the values typical for TPyB-Cu).

\section{Evolutionary Search for the ground state structures}

To analyze the ground state of the model using evolutionary approach, we used a Python package called DEAP [2-5] in conjunction with our SuSMoST code [6]. The DEAP package is open and has already established itself as an easy-to-use computing tool. The SuSMOST is also freely available and provides the ability to develop lattice models of two-dimensional adsorption layers and perform their statistical simulation, using a number of modern methods. Searching for lowenergy structures in the $\mathrm{TPyB}-\mathrm{Me} / \mathrm{Au}(111)$ model proposed in this work, we combined the capabilities of these two software packages. A simplified scheme of the evolutionary algorithm is shown in Figure S3. 


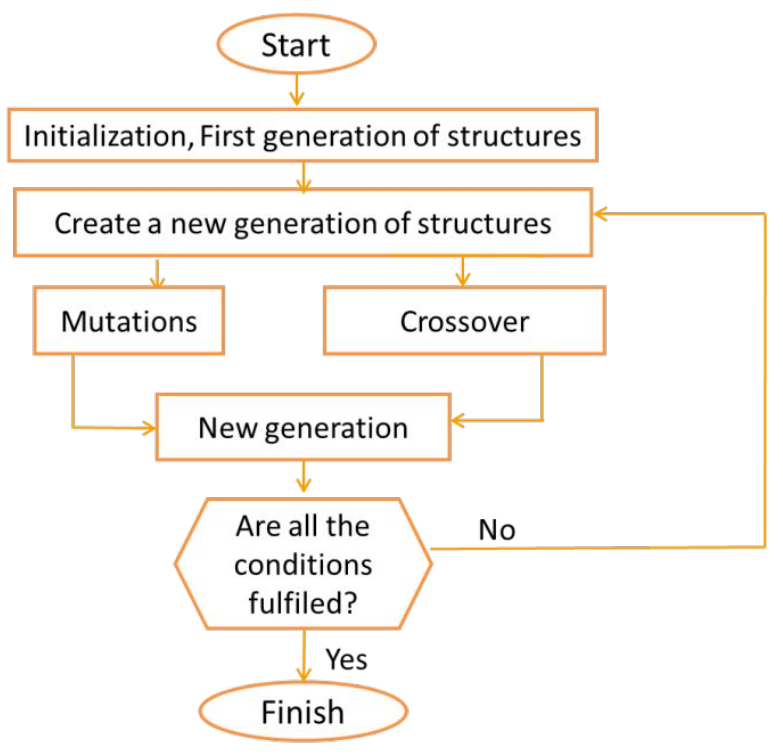

Figure S3. Simplified scheme of the evolutionary algorithm we used.

The TPyB- $\mathrm{Cu} / \mathrm{Au}(111)$ model is described in the main text. In evolutionary search for the low energy structures, an individual was presented as a list of integer numbers. The list length is determined by the number of adsorption sites on the simulation lattice. The list is filled with integers from 0 to 3 depending on the state of the lattice site: 0 is related to empty adsorption site, 1 and 2 - the adsorption site is occupied by TPyB molecule in one of the two possible orientations, 3 - the adsorption site is occupied by the copper atom (Figure S4).

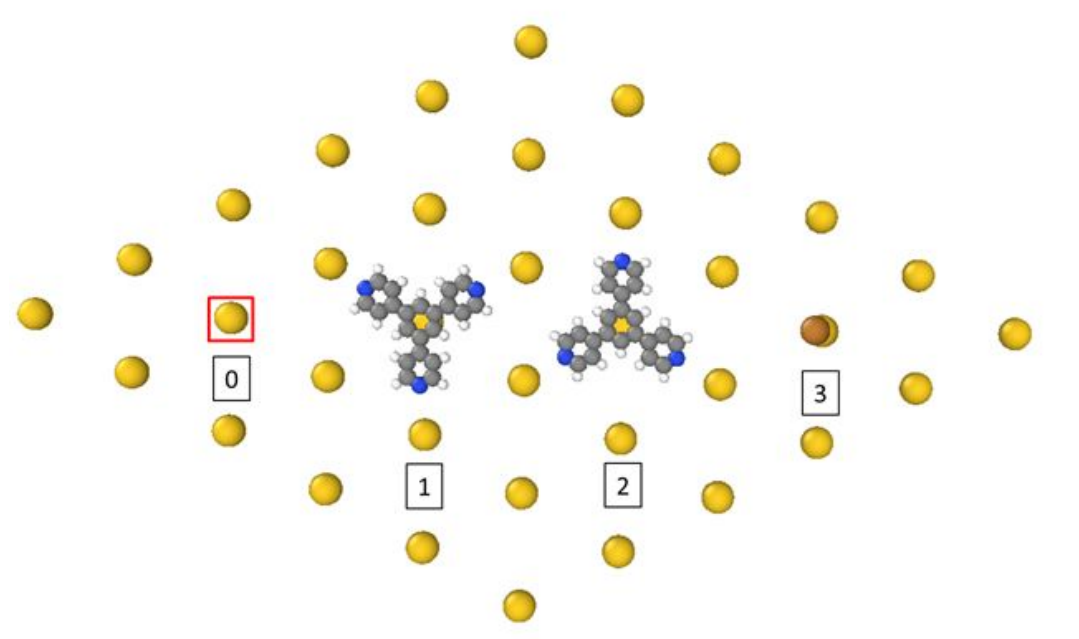


Figure S4. Possible states of the lattice site in our TPyB-Me/Au(111) model.

We set the lattice size $l \times l$ and the chemical potentials of the components $\mu_{T P y B}, \mu_{C u}$. Using native functions of the SuSMoST software package we have created the " $m$ " object, which is the lattice model in a format suitable for a statistical modeling in the SuSMoST code. Since we were interested only in the ground state of the model, the temperature was not used as a parameter of the evolutionary algorithm.

In order to prevent the forbidden configurations of the adsorption layer (for example, with overlapping neighboring molecules) in starting population, we used the "run" function of the SuSMoST code to create the initial generation (the "susmost_initialization" function). The function generates a random structure using 1000 Monte Carlo iterations. Examples of the first-generation structures are shown in Figure S5.

\section{def susmost_initialization():}

$\operatorname{run}\left(\mathrm{m}, \log \_\right.$periods_cnt $=1, \log \_$period_steps $=$None, $\log \_$callback $=$None, params_period_steps $=$ None, relaxation_steps $=1000$, bcast_full_params_log $=$ False, traj_fns=None)

individual $=\mathrm{m}$. cells $[:]$

return individual

Fitness factor has been defined as the total energy of the layer per lattice site. Fitness evaluation function is realized by two functions of the SuSMOST code: 
m.cells[:] = individual

m.recalc_curs()

return m.curE.sum(),

The "m.recalc_curs()" function recalculates current values of all the " $m$ " model parameters, in particular, total energy. The "m.curE.sum" function returns a current value of the system total energy.
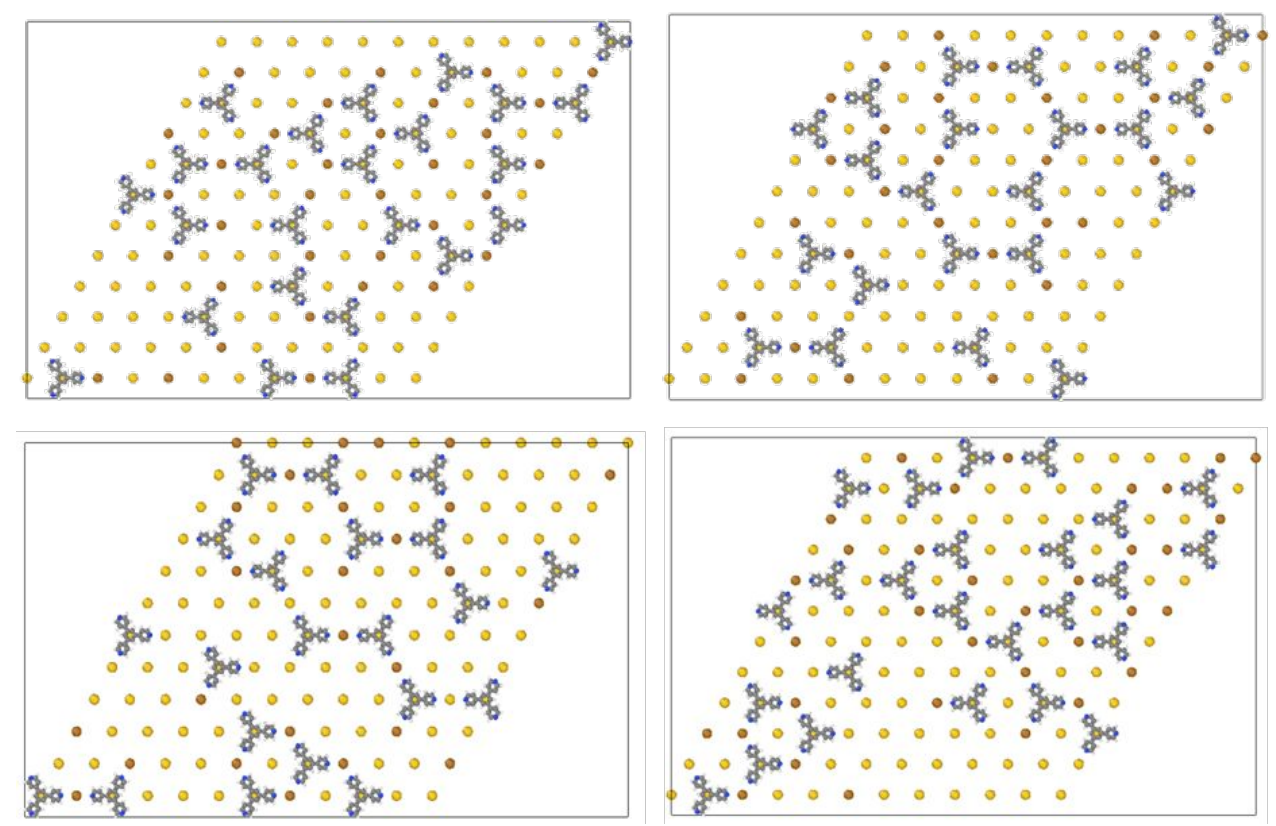

Figure S5. Few typical structures from a first generation.

Our goal was to find equilibrium structures, i.e. structures that have the least total energy (fitness function). We used the creator from the DEAP library to create a FitnessMin with weights $=(-1.0$,$) for fitness factor minimization:$

creator.create("FitnessMin", base.Fitness, weights=(-1.0,)) creator.create("Individual", list, fitness=creator.FitnessMin)

The population size was 300 individuals. At each step, 400 new individuals were generated using the crossover and mutation operators. The population was 
updated using the tournament selection (selTournament from the DEAP library) between 3 random chosen participants (tournsize). The tournament was attended by both newly acquired individuals and the old ones.

We used our "circular_mate" function as the crossover operator. In each of two randomly chosen individuals, it randomly selects two adsorption sites and determines their neighboring sites. Then, the states of the adsorption sites and their neighbors exchange between these individuals (Figure S6).
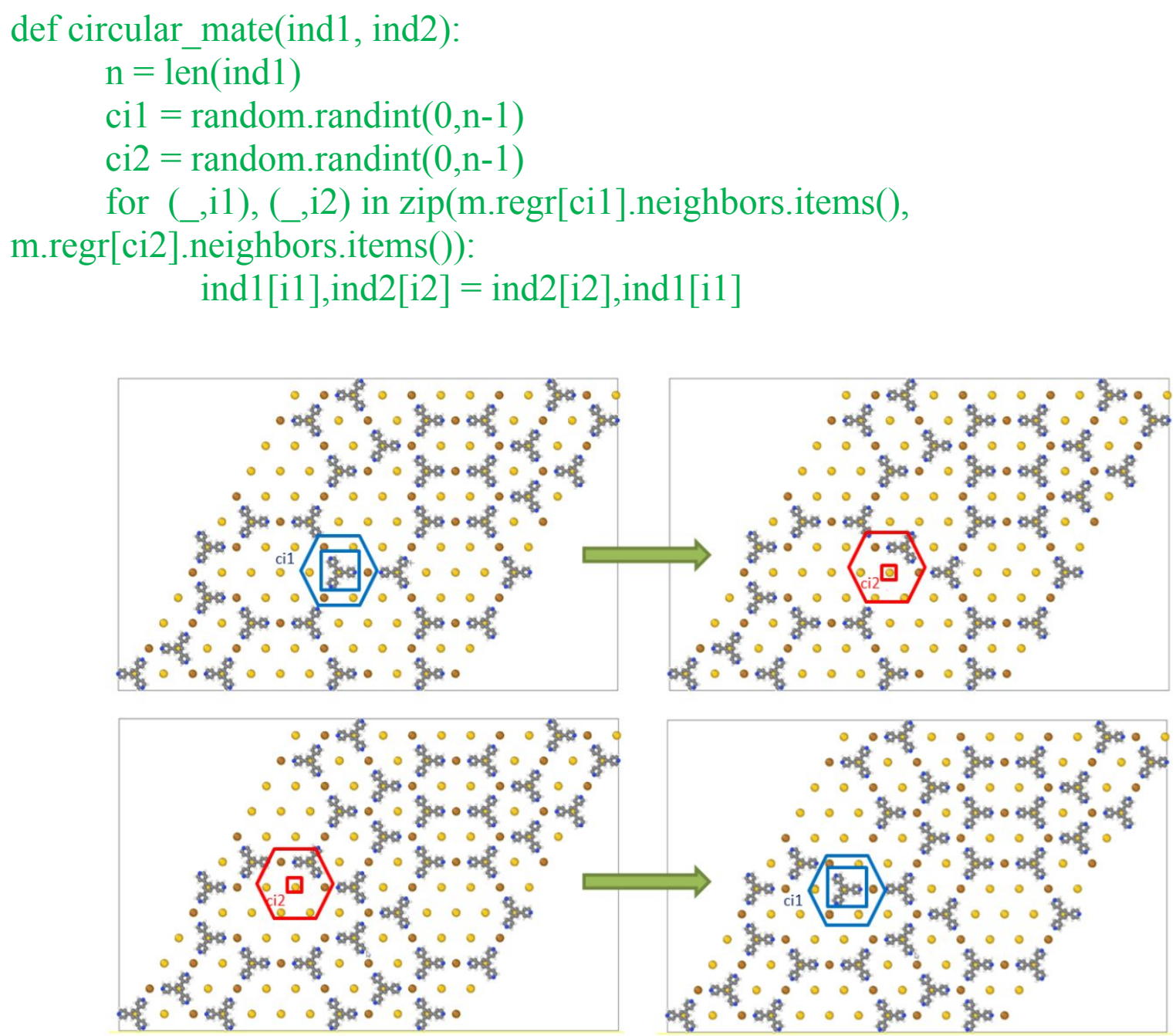

Figure S6. Scheme of the "circular_mate" crossover.

The ratio of the crossover probability to mutation probability was chosen 0.65: 0.35 . In the same ratio, we used two mutation operators: the Shuffle Indexes 
mutation (mutShuffleIndexes from the DEAP library) with a mutation probability of $1.4 \%$ and Monte Carlo mutation (one Monte Carlo step equaled to $l \times l$ iterations of the Metropolis algorithm). To maintain diversity in the population, we used the Fitness Sharing algorithm [7].

The algorithm performs 10,000 steps in total. Examples of the structures and energy convergence graphs obtained during the evolutionary search are presented in Figure S7 and Figure S8. During the evolutionary search both structures from the homologous series of flower phases and alternative low-energy structures were obtained. The last ones may exist in the $\mathrm{TPyB}-\mathrm{Cu} / \mathrm{Au}(111)$ layer due to kinetic difficulties in the self-assembly of the equilibrium structures. Such combination of the deterministic and evolutionary approaches in the form of the sequential iterative scheme makes the ground state analysis of the model much more reliable. 

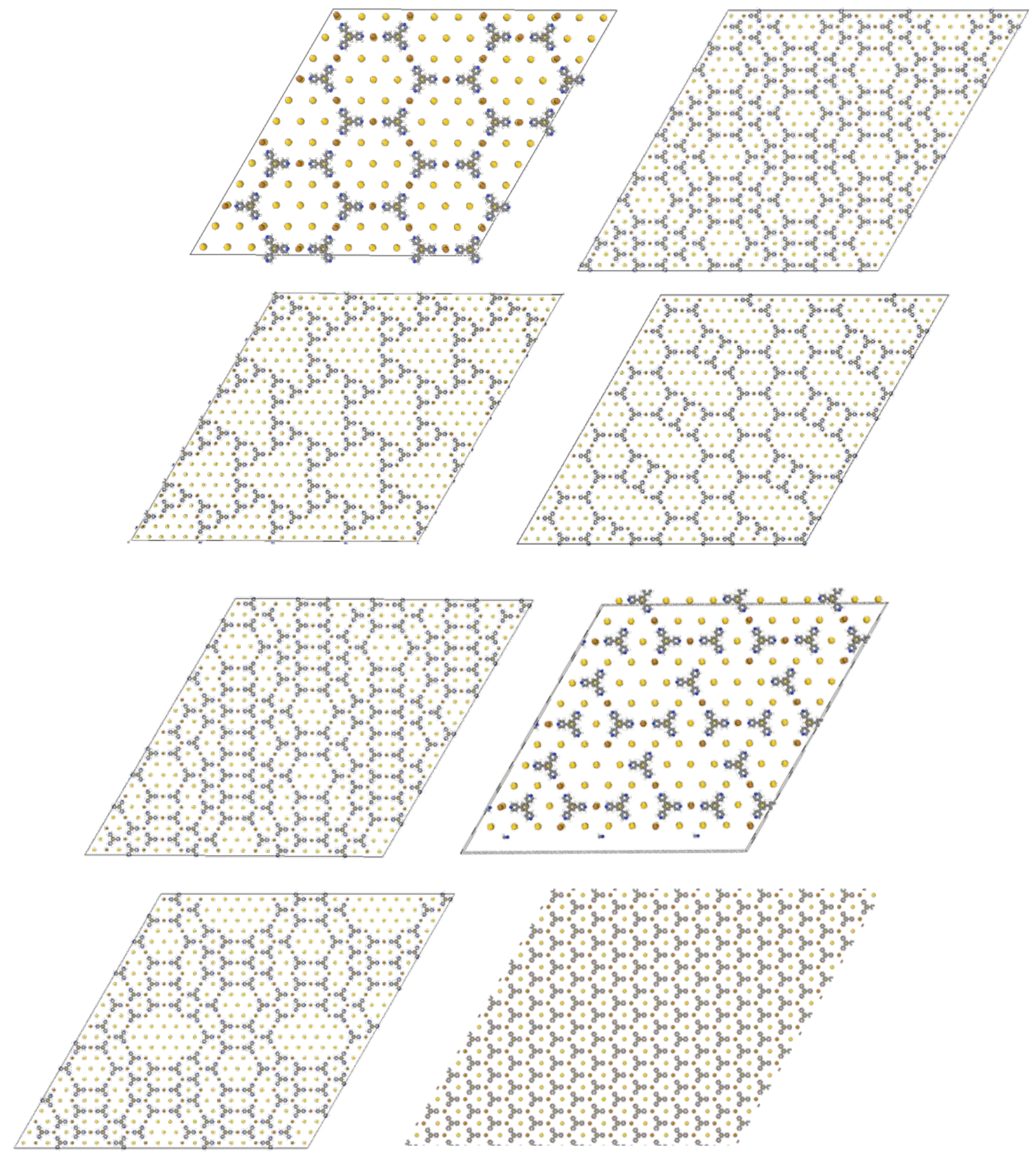

Figure S7. Examples of metal-organic structures in the TPyB-Cu/Au(111) layer that we have found using the evolutionary algorithm. 

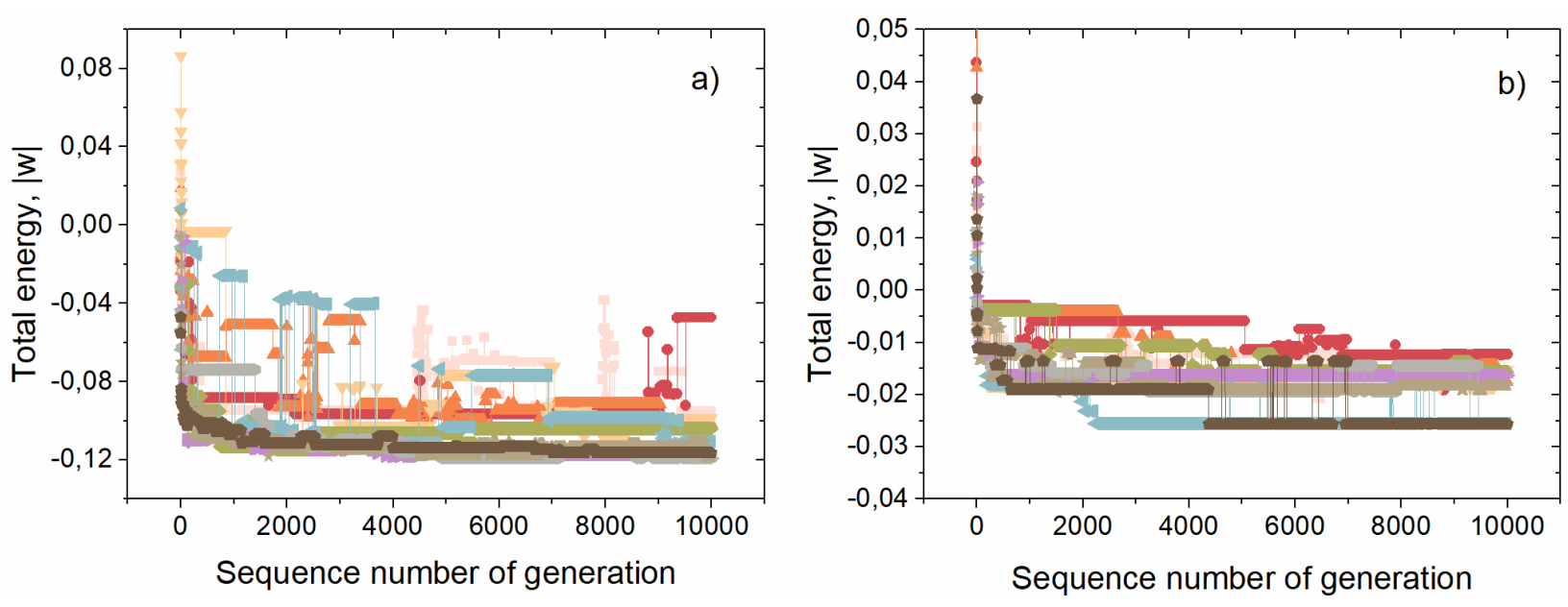

Figure S8. Examples of the energy convergence curves along the evolutionary search: a) for the honeycomb structure, b) for the flower-like structure.

\section{Some results of tensor renormalization group calculations}

Using TRG method we have also calculated the entropy and heat capacity of the TPyB-Cu/Au(111) adsorption layer (Figure S9).

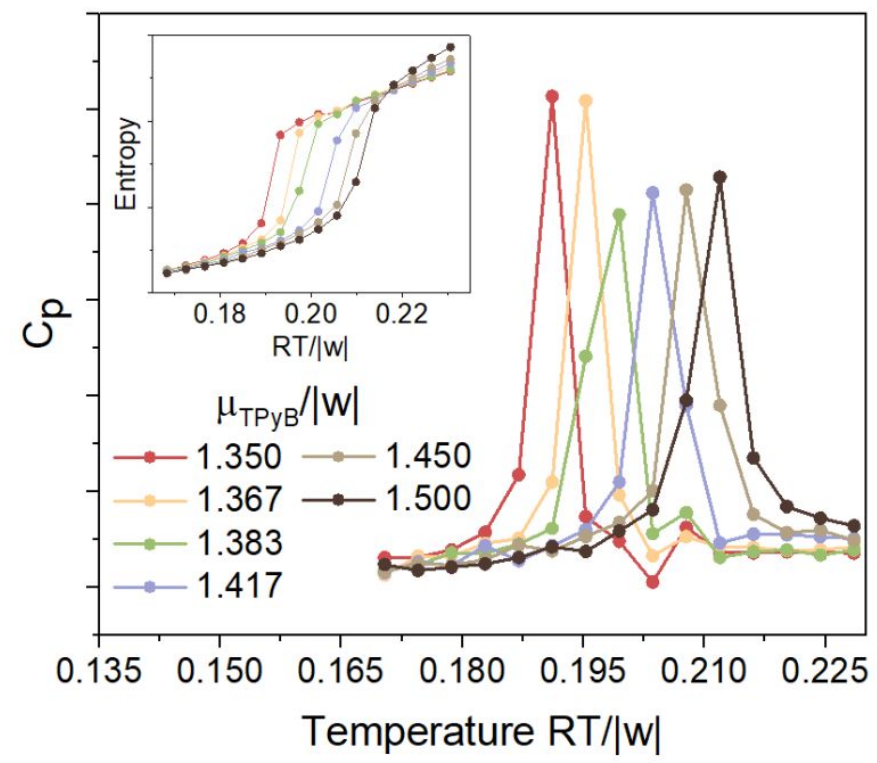

Figure S9. Heat capacity of the $\mathrm{TPyB}-\mathrm{Cu} / \mathrm{Au}(111)$ model layer vs. temperature calculated by the TRG algorithm at specified values of the $\mathrm{Cu}$ chemical potential. 
On the inset: the corresponding dependencies of the entropy of the adsorption layer on temperature.

The entropy of the layer was computed by numeric differentiation of the grand thermodynamic potential obtained directly in the TRG calculations with respect to temperature. Second derivative gives us the heat capacity [8].

\section{DFT calculations}

To calculate the ratio between two-fold and three-fold coordination modes we used the following formulas:

$$
\begin{aligned}
& \omega_{d}=E_{2 T P y B}-M e-2 \cdot E_{T P y B}-E_{M e}, \\
& \omega_{t}=E_{3 T P y B}-M e-3 \cdot E_{T P y B}-E_{M e},
\end{aligned}
$$

where $E_{2 T P Y B-M e}$ and $E_{3 T P y B}-M e$ - total energy of the system including one metal atom and two or three TPyB molecules, respectively. Figure S10 demonstrates an example of such configurations for the copper atom. $E_{T P y B}$ and $E_{M e}$ - total energy of the isolated TPyB molecule and metal atom.

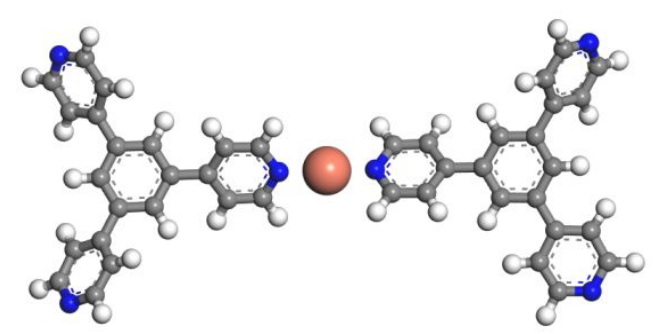




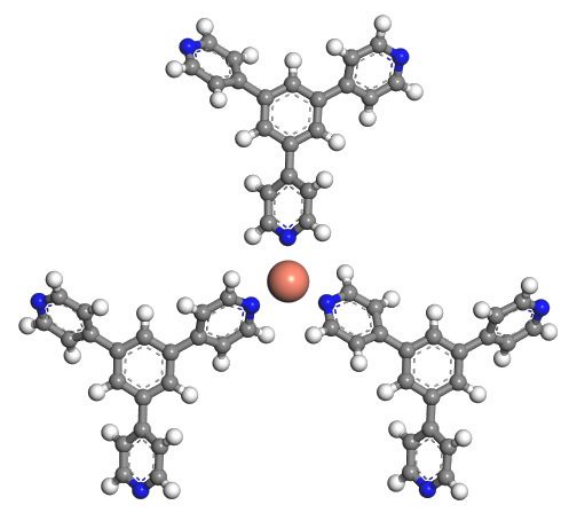

Figure S10. Two-fold and three-fold TPyB-Cu coordination as it results from the DFT-GGA/PBE calculations, using dispersion correction (DFT-D3) and the basis set with valence triple-zeta polarization (def2-TZVP).

We have calculated the TPyB-Me two-fold and three-fold coordination energies for all combinations of the oxidation states of the selected metals (Table S1). The sequence $\mathrm{Ag} \leq \mathrm{Cu}<\mathrm{Ni}<\mathrm{Co}<\mathrm{Fe}$ is maintained as long as the oxidation state of the metal coordinating center in the three-fold configuration is less than or equal to its oxidation state in the two-fold configuration. The larger the difference between the oxidation states in the two-fold and three-fold coordination motifs, the smaller the ratio $w_{t} / w_{d}$.

Table S1. Ratio between the Energies of TPyB-Me Binding Modes $w_{t} / w_{d}$ Obtained by DFT Calculations for Various Transition Metals and Combination of the Possible Oxidation States

\begin{tabular}{ccccc}
\hline & \multicolumn{4}{c}{$\boldsymbol{w}_{\boldsymbol{t}} / \boldsymbol{w}_{\boldsymbol{d}}$} \\
Oxidation state $(+)$ & $\mathbf{0 / 0}$ & $\mathbf{0 / 1}$ & $\mathbf{0 / 2}$ & $\mathbf{0 / 3}$ \\
\hline $\mathbf{A g}$ & 1.2386 & 0.364696 & 0.101461 & - \\
$\mathbf{C u}$ & 1.2141 & 0.508883 & 0.171812 & - \\
$\mathbf{N i}$ & 1.4264 & 0.709983 & 0.244018 & 0.096576 \\
$\mathbf{C o}$ & 1.4314 & 0.724153 & 0.24359 & 0.097984 \\
$\mathbf{F e}$ & 1.5196 & 0.858792 & 0.265004 & 0.106686
\end{tabular}




\begin{tabular}{ccccc} 
Oxidation state (+) & $\mathbf{1 / 0}$ & $\mathbf{1 / 1}$ & $\mathbf{1 / 2}$ & $\mathbf{1 / 3}$ \\
\hline $\mathbf{A g}$ & 3.819196 & 1.124572 & 0.312864 & - \\
$\mathbf{C u}$ & 2.705548 & 1.134032 & 0.382879 & - \\
$\mathbf{N i}$ & 2.549328 & 1.268881 & 0.436108 & 0.172601 \\
$\mathbf{C o}$ & 2.619201 & 1.325068 & 0.445726 & 0.179293 \\
$\mathbf{F e}$ & 2.571099 & 1.45304 & 0.448375 & 0.180508 \\
Oxidation state (+) & $\mathbf{2 / 0}$ & $\mathbf{2 / 1}$ & $\mathbf{2 / 2}$ & $\mathbf{2 / 3}$ \\
\hline $\mathbf{A g}$ & 12.83949 & 3.780622 & 1.051796 & - \\
$\mathbf{C u}$ & 7.519878 & 3.151961 & 1.064185 & - \\
$\mathbf{N i}$ & 6.510379 & 3.240421 & 1.113717 & 0.440782 \\
$\mathbf{C o}$ & 6.572919 & 3.325276 & 1.118555 & 0.449939 \\
$\mathbf{F e}$ & 6.565443 & 3.710417 & 1.144951 & 0.460936 \\
Oxidation state (+) & $\mathbf{3 / 0}$ & $\mathbf{3 / 1}$ & $\mathbf{3 / 2}$ & $\mathbf{3 / 3}$ \\
\hline $\mathbf{A g}$ & - & - & - & - \\
$\mathbf{C u}$ & - & - & - & - \\
$\mathbf{N i}$ & 15.68456 & 7.806702 & 2.683126 & 1.061915 \\
$\mathbf{C o}$ & 15.59012 & 7.887129 & 2.65307 & 1.067197 \\
$\mathbf{F e}$ & 15.37099 & 8.686815 & 2.680555 & 1.079142 \\
\hline
\end{tabular}

As it can be seen, when the oxidation state of the metal center in three-fold coordination motif is larger than two-fold one, the $w_{t} / w_{d}$ ratio is more than 2 for all the metals. It means that all the considered $\mathrm{TPyB}-\mathrm{Me} / \mathrm{Au}(111)$ layers tend to form only the triangular structure, including the $\mathrm{TPyB}-\mathrm{Cu} / \mathrm{Au}(111)$ layer. It obviously contradicts the experimental data. The same is true, when oxidation states of the metals in three-fold and two-fold configurations differ by more than one. In this case, the $w_{t} / w_{d}$ ratio is sufficiently less than one, therefore the honeycomb structure should dominate for all the TPyB-Me/Au(111) layers. But it doesn't, as it follows from the published data. For these reasons we used the same oxidation states of the metal centers in the two-fold and three-fold coordination motifs in our DFT calculations of the $w_{t} / w_{d}$ ratio.

\section{REFERENCES}


1. Liu, J.; Lin, T.; Shi, Z.; Xia, F.; Dong, L.; Liu, P. N.; Lin, N. Structural Transformation of Two-

Dimensional Metal-Organic Coordination Networks Driven by Intrinsic In-Plane Compression. J.

Am. Chem. Soc. 2011, 133, 18760-18766.

2. https://github.com/deap/deap

3. Fortin, F.-A.; De Rainville, F.-M.; Gardner, M.-A.; Parizeau, M.; Gagné, C. DEAP: Evolutionary Algorithms Made Easy. Journal of Machine Learning Research 2012, 13, 21712175.

4. De Rainville, F.-M.; Fortin, F-A.; Gardner, M-A.; Parizeau, M.; Gagné, C. DEAP: A python framework for evolutionary algorithms. Proceedings of the 14th annual conference companion on Genetic and evolutionary computation. 2012. 85-92.

\section{https://doi.org/10.1145/2330784.2330799}

5. Kim, J.; Yoo, S. Software review: DEAP (Distributed Evolutionary Algorithm in Python) library // Genetic Program and Evolvable Machines 2019, 20. 139-142.

6. http://susmost.com/

7. A.E. Eiben, James E. Smith, Introduction to Evolutionary Computing, Springer-Verlag Berlin Heidelberg, 2015, 287 p.

8. Akimenko, S. S.; Gorbunov, V. A.; Myshlyavtsev, A. V.; Stishenko, P. V. Tensor renormalization group study of hard-disk models on a triangular lattice. Phys. Rev. E. 2019, 100, 022108 . 